CRYSTALLOGRAPHIC COMMUNICATIONS

ISSN 2056-9890

Received 11 April 2017

Accepted 17 April 2017

Edited by M. Weil, Vienna University of Technology, Austria

Keywords: crystal structure; 1,4,8,11-tetraazoniacyclotetradecane; dichromate anion; hydrogen bonding; synchrotron radiation.

CCDC reference: 1534082

Supporting information: this article has supporting information at journals.iucr.org/e

\section{Crystal structure of 1,4,8,11-tetraazoniacyclotetra- decane bis(dichromate) monohydrate from synchrotron data}

\author{
Dohyun Moon ${ }^{\mathrm{a}}$ and Jong-Ha Choi ${ }^{\mathrm{b} *}$
}

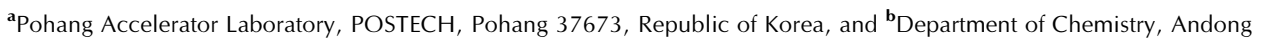
National University, Andong 36729, Republic of Korea. *Correspondence e-mail: jhchoi@anu.ac.kr

The asymmetric unit of the hydrated title salt, $\left(\mathrm{C}_{10} \mathrm{H}_{28} \mathrm{~N}_{4}\right)\left[\mathrm{Cr}_{2} \mathrm{O}_{7}\right]_{2} \cdot \mathrm{H}_{2} \mathrm{O}$ $\left[\mathrm{C}_{10} \mathrm{H}_{28} \mathrm{~N}_{4}=\mathrm{H}_{4}(\right.$ cyclam $)=1,4,8,11$-tetraazoniacyclotetradecane], contains two half-cations (both completed by crystallographic inversion symmetry), two dichromate anions and one water molecule. The two $\left[\mathrm{CrO}_{7}\right]^{2-}$ anions exhibit a nearly staggered conformation, with bridging angles of 133.37 (11) and $136.28(12)^{\circ}$. The distortions of the dichromate anions are due to their participation in hydrogen-bonding interactions with the water molecule and the cations. Intermolecular hydrogen bonds involving the cyclam $\mathrm{N}-\mathrm{H}$ groups and water $\mathrm{O}-\mathrm{H}$ groups as donor groups, and the $\mathrm{O}$ atoms of the dichromate anions as acceptor groups give rise to a three-dimensional network.

\section{Chemical context}

Chromium(VI) compounds are highly cytotoxic and potential carcinogens (Cohen et al., 1993). A number of treatment methods for the removal of such toxic heavy metal ions in water have been described (Kalidhasan et al., 2016), and 1,4,8,11-tetraazacyclotetradecane (cyclam) is possibly one of the most useful candidates for this purpose since it has a strong ability to act as an effective metal-ion binding molecule. The azamacrocycle is a strong basic amine to form a dication, $\left(\mathrm{C}_{10} \mathrm{H}_{26} \mathrm{~N}_{4}\right)^{2+}$, or a tetracation, $\left(\mathrm{C}_{10} \mathrm{H}_{28} \mathrm{~N}_{4}\right)^{4+}$, in both of which all of the $\mathrm{N}-\mathrm{H}$ bonds are generally active in hydrogen-bond formation. These di- or tetraammonium cations may also be suitable candidates for the removal of toxic metal ions. Previously, the syntheses and crystal structures of $\left[\mathrm{H}_{2}\right.$ (cyclam) $]\left(\mathrm{ClO}_{4}\right)_{2}$ (Nave \& Truter, 1974), $\left[\mathrm{H}_{2}\right.$ (cyclam) $] \mathrm{Cl}_{2} \cdot$ $0.5 \mathrm{H}_{2} \mathrm{O}$ (Kim et al., 2009), $\left[\mathrm{H}_{4}(\right.$ cyclam $\left.)\right]\left(\mathrm{NO}_{3}\right)_{4} \cdot 2 \mathrm{H}_{2} \mathrm{O}$ (Harrowfield et al., 1996), $\left[\mathrm{H}_{2}\right.$ (cyclam)](maleate) 2 (Mireille Ninon et al., 2013), $\left[\mathrm{H}_{4}(\mathrm{cyclam})\right]\left(\mathrm{HSO}_{4}\right)_{4}$ (Said et al., 2013), $\left[\mathrm{H}_{4}(\right.$ cyclam $\left.)\right] \mathrm{Cl}_{4}, \quad\left[\mathrm{H}_{4}(\right.$ cyclam $\left.)\right] \mathrm{Cl}_{4} \cdot 4 \mathrm{H}_{2} \mathrm{O}, \quad\left[\mathrm{H}_{4}(\right.$ cyclam $\left.)\right] \mathrm{Br}_{4} \cdot-$ $4 \mathrm{H}_{2} \mathrm{O},\left[\mathrm{H}_{4}\right.$ (cyclam) $]\left(\mathrm{ClO}_{4}\right)_{4} \cdot 2 \mathrm{H}_{2} \mathrm{O}$ (Robinson et al., 1989) and $\left[\mathrm{H}_{4}(\right.$ cyclam $\left.)\right]\left(\mathrm{SO}_{4}\right)_{2} \cdot 6 \mathrm{H}_{2} \mathrm{O}$ (Subramanian \& Zaworotko, 1995) have been reported. The crystal structure of neutral cyclam has also been determined (Robinson et al., 1989), but a combination of the 1,4,8,11-tetraazoniacyclotetradecane cation with the $\left[\mathrm{CrO}_{7}\right]^{2-}$ anion has not been reported. We give here details of the preparation of the title compound, a new hydrated organic dichromate(VI) salt, $\left[\mathrm{H}_{4}(\right.$ cyclam $\left.)\right]\left[\mathrm{Cr}_{2} \mathrm{O}_{7}\right]_{2} \cdot-$ $\mathrm{H}_{2} \mathrm{O}$, (I), and its structural characterization by synchrotron single-crystal X-ray diffraction. 


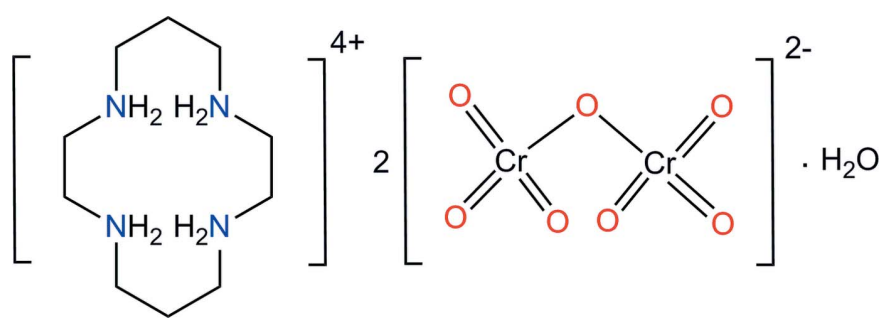

\section{Structural commentary}

An ellipsoid plot of the molecular components of (I) along with the atom-numbering scheme is shown in Fig. 1. The asymmetric unit comprises of two half-cations (both completed by crystallographic inversion symmetry), two dichromate anions and one water molecule. Within the centrosymmetric tetra-protonated amine unit, $\left(\mathrm{C}_{10} \mathrm{H}_{28} \mathrm{~N}_{4}\right)^{4+}$, the $\mathrm{C}-\mathrm{C}$ and $\mathrm{N}-\mathrm{C}$ bond lengths range from 1.491 (3) to 1.520 (3) $\AA$ and from 1.489 (3) to 1.524 (3) $\AA$, respectively. The range of $\mathrm{N}-\mathrm{C}-\mathrm{C}$ and $\mathrm{C}-\mathrm{N}-\mathrm{C}$ angles is 109.84 (19) to $116.69(18)^{\circ}$ and $110.15(18)$ to $111.5(2)^{\circ}$, respectively. Bond lengths and angles within the tetraammonium cations are comparable to the corresponding values determined for the cyclam ligand in trans- $\left[\mathrm{Cr}(\text { nic-O })_{2}(\right.$ cyclam $\left.)\right] \mathrm{ClO}_{4}($ nic-O $=\mathrm{O}-$ coordinating nicotinate; Choi, 2009), cis- $\left[\mathrm{Cr}(\mathrm{ONO})_{2}\right.$ (cyclam $)] \mathrm{NO}_{2}$ (Choi et al., 2004a), $[\mathrm{Cr}(\mathrm{ox})($ cyclam $)] \mathrm{ClO}_{4}(\mathrm{ox}=$ oxalate; Choi et al., 2004b), [Cr(acac)(cyclam) $\left(\mathrm{ClO}_{4}\right)_{2} \cdot 0.5 \mathrm{H}_{2} \mathrm{O}$ (acac = acetylacetonate; Subhan et al., 2011), cis$\left[\mathrm{Cr}(\mathrm{NCS})_{2}(\right.$ cyclam) $] \mathrm{NCS}$ (Moon et al., 2013) or $\left[\mathrm{CrCl}_{2}\right.$ (cyclam) $][\mathrm{Cr}(\mathrm{ox})($ cyclam $)]\left(\mathrm{ClO}_{4}\right)_{2}$ (Moon \& Choi, 2016).

It is of interest to compare the conformation of the $\left[\mathrm{CrO}_{7}\right]^{2-}$ anion with those found in other ionic crystals. In (I), the two $\left[\mathrm{CrO}_{7}\right]^{2-}$ anions exhibit a nearly staggered conformation whereas an eclipsed conformation is observed for $\left(\mathrm{C}_{3} \mathrm{H}_{5} \mathrm{~N}_{2}\right)\left(\mathrm{NH}_{4}\right)\left[\mathrm{Cr}_{2} \mathrm{O}_{7}\right]$ or $\left(\mathrm{C}_{9} \mathrm{H}_{14} \mathrm{~N}\right)_{2}\left[\mathrm{Cr}_{2} \mathrm{O}_{7}\right]$ (Zhu, 2012;

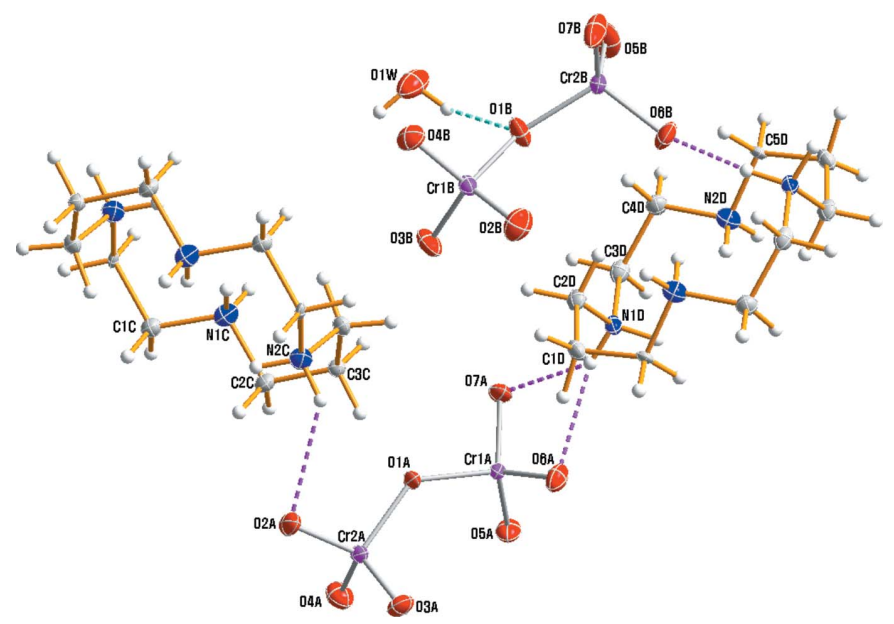

Figure 1

The structures of the molecular components in (I), drawn with displacement ellipsoids at the $60 \%$ probability level. Dashed lines represent hydrogen-bonding interactions.
Table 1

Hydrogen-bond geometry $\left(\AA,^{\circ}\right)$.

\begin{tabular}{lllll}
\hline$D-\mathrm{H} \cdots A$ & $D-\mathrm{H}$ & $\mathrm{H} \cdots A$ & $D \cdots A$ & $D-\mathrm{H} \cdots A$ \\
\hline $\mathrm{N} 1 C-\mathrm{H} 1 N C \cdots \mathrm{O} 2 B^{\mathrm{i}}$ & 0.91 & 2.45 & $3.091(3)$ & 128 \\
$\mathrm{~N} 1 D-\mathrm{H} 1 N D \cdots \mathrm{O} 6 A$ & 0.91 & 2.38 & $3.140(3)$ & 142 \\
$\mathrm{~N} 1 D-\mathrm{H} 1 N D \cdots \mathrm{O} 7 A$ & 0.91 & 2.16 & $2.942(3)$ & 143 \\
$\mathrm{~N} 1 D-\mathrm{H} 2 N D \cdots \mathrm{O} 6 B^{\mathrm{ii}}$ & 0.91 & 1.87 & $2.768(3)$ & 169 \\
$\mathrm{~N} 2 C-\mathrm{H} 3 N C \cdots \mathrm{O} 6 A^{\mathrm{iii}}$ & 0.91 & 2.62 & $3.074(3)$ & 112 \\
$\mathrm{~N} 2 C-\mathrm{H} 4 N C \cdots \mathrm{O} B^{\mathrm{iv}}$ & 0.91 & 2.42 & $3.047(3)$ & 126 \\
$\mathrm{~N} 2 C-\mathrm{H} 4 N C \cdots \mathrm{O} 2 A$ & 0.91 & 2.52 & $3.200(3)$ & 132 \\
$\mathrm{~N} 2 D-\mathrm{H} 3 N D \cdots \mathrm{O} 6 B^{\mathrm{ii}}$ & 0.91 & 2.42 & $3.198(3)$ & 144 \\
$\mathrm{~N} 2 D-\mathrm{H} 4 N D \cdots \mathrm{O} 2 B^{\mathrm{ii}}$ & 0.91 & 2.65 & $3.357(3)$ & 136 \\
$\mathrm{O} 1 W-\mathrm{H} 1 O 1 \cdots \mathrm{O} 5 A^{\mathrm{v}}$ & $0.84(1)$ & $2.38(10)$ & $2.999(3)$ & $130(11)$ \\
$\mathrm{O} 1 W-\mathrm{H} 2 O 1 \cdots \mathrm{O} 1 B$ & $0.84(1)$ & $2.05(4)$ & $2.774(3)$ & $143(5)$ \\
\hline
\end{tabular}

Symmetry codes: (i) $x+1, y, z$; (ii) $-x+1,-y+1,-z+1$; (iii) $x,-y+\frac{1}{2}, z-\frac{1}{2}$; (iv) $-x+1, y-\frac{1}{2},-z+\frac{1}{2} ;(\mathrm{v})-x+2, y+\frac{1}{2},-z+\frac{1}{2}$.

Trabelsi et al., 2015). The conformation of dichromate anions appears to show a dependence on the size of the associated counter-cation (Moon et al., 2015, 2017). The $\mathrm{Cr} 1 A-\mathrm{O} 1 A-$ $\mathrm{Cr} 2 A$ and $\mathrm{Cr} 1 B-\mathrm{O} 1 B-\mathrm{Cr} 2 B$ bridging angles in the anions in (I) are $133.37(11)$ and $136.28(12)^{\circ}$, respectively, slightly larger than $130.26(10)^{\circ}$ in $\left[\mathrm{Cr}(\text { urea })_{6}\right]\left[\mathrm{Cr}_{2} \mathrm{O}_{7}\right] \mathrm{Br} \cdot \mathrm{H}_{2} \mathrm{O}$ (Moon et al., 2015). The smaller $\mathrm{Cr} 1 A-\mathrm{O} 1 A-\mathrm{Cr} 2 A$ bridging angle is probably due to the non-involvement of the terminal oxygen atoms of $\mathrm{Cr} 2 A$ in any hydrogen bond. $\mathrm{Cr}-\mathrm{O}_{\mathrm{b}}\left(\mathrm{O}_{\mathrm{b}}=\right.$ bridging O atom) bonds range from 1.7711 (19) to 1.799 (2) A while the $\mathrm{Cr}-\mathrm{O}_{\mathrm{t}}$ bond lengths to the terminal $\mathrm{O}$ atoms vary from 1.590 (2) to 1.6417 (19) $\AA$, with a mean terminal $\mathrm{Cr}-\mathrm{O}$ bond length of $1.615 \AA$. The $\mathrm{Cr}-\mathrm{O}$ bond lengths for atoms involved in hydrogen-bonding interactions are slightly longer than the other $\mathrm{Cr}-\mathrm{O}$ bonds. This trend is similar to that observed for



Figure 2

The crystal packing in compound (I), viewed perpendicular to the $b c$ plane. Dashed lines represent $\mathrm{N}-\mathrm{H} \cdots \mathrm{O}$ (pink) and $\mathrm{O}-\mathrm{H} \cdots \mathrm{O}$ (cyan) hydrogen-bonding interactions, respectively. $\mathrm{H}$ atoms bound to $\mathrm{C}$ atoms have been omitted. 
comparable anions in the structures of [ $\left.\mathrm{Cr}(\text { urea })_{6}\right]\left[\mathrm{Cr}_{2} \mathrm{O}_{7}\right]-$ $\mathrm{Br} \cdot \mathrm{H}_{2} \mathrm{O}$ (Moon et al., 2015), $\left[\mathrm{Cr}(\mathrm{NCS})_{2}(\text { cyclam) }]_{2}\left[\mathrm{Cr}_{2} \mathrm{O}_{7}\right] \cdot \mathrm{H}_{2} \mathrm{O}\right.$ (Moon et al., 2017) or $\left[\mathrm{Cr}(\right.$ ox $)(\text { cyclam) }]_{2}\left[\mathrm{Cr}_{2} \mathrm{O}_{7}\right] \cdot 8 \mathrm{H}_{2} \mathrm{O}$ (Moon \& Choi, 2017).

\section{Supramolecular features}

Extensive $\mathrm{N}-\mathrm{H} \cdots \mathrm{O}$ and $\mathrm{O}-\mathrm{H} \cdots \mathrm{O}$ hydrogen-bonding interactions occur in the crystal structure (Table 1). Two $\mathrm{O}-$ $\mathrm{H}$... O hydrogen bonds link the water molecule to two neighboring $\left[\mathrm{CrO}_{7}\right]^{2-}$ anions while $\mathrm{N}-\mathrm{H} \cdots \mathrm{O}$ hydrogen bonds interconnect the $\left(\mathrm{C}_{10} \mathrm{H}_{28} \mathrm{~N}_{4}\right)^{4+}$ cations with both anions (Figs. 1 and 2). An extensive array of these contacts generates a three-dimensional network (Fig. 2) and, apart from Coulombic interactions, these hydrogen-bonding interactions help to stabilize the crystal structure.

\section{Database survey}

A search of the Cambridge Structural Database (Version 5.38, Feb 2017 with two updates; Groom et al. 2016) revealed a total of 24 hits for compounds containing 1,4,8,11-tetraazoniacyclotetradecane $\left(\mathrm{C}_{10} \mathrm{H}_{28} \mathrm{~N}_{4}\right)^{4+}$ or 4,11-diaza-1,8-diazoniacyclotetradecane $\left(\mathrm{C}_{10} \mathrm{H}_{26} \mathrm{~N}_{4}\right)^{2+}$ cations, but a combination with dichromate anions has not been reported.

\section{Synthesis and crystallization}

Cyclam (98\%) was purchased from Sigma-Aldrich and used without further purification. All other chemicals were reagentgrade materials, and were used as received. $0.102 \mathrm{~g}$ of chromium trioxide (1 mmol, Sigma-Aldrich) was dissolved in $20 \mathrm{ml}$ of water and $0.012 \mathrm{~g}$ of cyclam $(0.06 \mathrm{mmol}$, SigmaAldrich) was added at room temperature. The mixture was stirred for 30 minutes and the resulting solution was filtered. The neat filtrate was allowed to stand for one week to give block-like yellow crystals of (I) suitable for X-ray structural analysis.

\section{Refinement}

Crystal data, data collection and structure refinement details are summarized in Table 2. All hydrogen atoms were placed in geometrically idealized positions and constrained to ride on their parent atoms, with $\mathrm{C}-\mathrm{H}=0.99 \AA$ and $\mathrm{N}-\mathrm{H}=0.91 \AA$, respectively, and with $U_{\text {iso }}(\mathrm{H})$ values of $1.2 U_{\text {eq }}$ of the parent atoms. The hydrogen atoms of the solvent water molecule were assigned based on a difference-Fourier map, and were refined with distance restraints of 0.84 (2) A (using DFIX and DANG commands), and with $U_{\text {iso }}(\mathrm{H})$ values of $1.5 U_{\text {eq }}$ of the parent atom. The remaining maximum and minimum electron densities in the final Fourier map are located 0.85 and $0.54 \AA$, respectively, from the $\mathrm{Cr} 1 B$ site. Six reflections with a poor agreement between measured and calculated intensities were omitted from the final refinement cycles.
Table 2

Experimental details.

\begin{tabular}{|c|c|}
\hline \multicolumn{2}{|l|}{ Crystal data } \\
\hline Chemical formula & $\left(\mathrm{C}_{10} \mathrm{H}_{28} \mathrm{~N}_{4}\right)\left[\mathrm{Cr}_{2} \mathrm{O}_{7}\right]_{2} \cdot \mathrm{H}_{2} \mathrm{O}$ \\
\hline$M_{\mathrm{r}}$ & 654.38 \\
\hline Crystal system, space group & Monoclinic, $P 2_{1} / c$ \\
\hline Temperature $(\mathrm{K})$ & 200 \\
\hline$a, b, c(\AA)$ & $10.428(2), 13.961(2), 15.490(2)$ \\
\hline$\beta\left(^{\circ}\right)$ & $94.671(3)$ \\
\hline$V\left(\AA^{3}\right)$ & $2247.6(6)$ \\
\hline$Z$ & 4 \\
\hline Radiation type & Synchrotron, $\lambda=0.610 \AA$ \\
\hline$\mu\left(\mathrm{mm}^{-1}\right)$ & 1.28 \\
\hline Crystal size $(\mathrm{mm})$ & $0.11 \times 0.10 \times 0.09$ \\
\hline \multicolumn{2}{|l|}{ Data collection } \\
\hline Diffractometer & ADSC Q210 CCD area detector \\
\hline Absorption correction & $\begin{array}{l}\text { Empirical (using intensity } \\
\text { measurements) (HKL3000sm } \\
\text { SCALEPACK; Otwinowski \& } \\
\text { Minor, 1997) }\end{array}$ \\
\hline$T_{\min }, T_{\max }$ & $0.942,1.000$ \\
\hline $\begin{array}{l}\text { No. of measured, independent and } \\
\text { observed }[I>2 \sigma(I)] \text { reflections }\end{array}$ & $12953,6614,5804$ \\
\hline$R_{\text {int }}$ & 0.016 \\
\hline$(\sin \theta / \lambda)_{\max }\left(\AA^{-1}\right)$ & 0.706 \\
\hline \multicolumn{2}{|l|}{ Refinement } \\
\hline$R\left[F^{2}>2 \sigma\left(F^{2}\right)\right], w R\left(F^{2}\right), S$ & $0.038,0.124,1.09$ \\
\hline No. of reflections & 6614 \\
\hline No. of parameters & 307 \\
\hline No. of restraints & 3 \\
\hline $\mathrm{H}$-atom treatment & $\begin{array}{l}\mathrm{H} \text { atoms treated by a mixture of } \\
\text { independent and constrained } \\
\text { refinement }\end{array}$ \\
\hline$\Delta \rho_{\max }, \Delta \rho_{\min }\left(\mathrm{e} \AA^{-3}\right)$ & $2.21,-1.04$ \\
\hline
\end{tabular}

Computer programs: PAL BL2D-SMDC (Shin et al., 2016), HKL3000sm (Otwinowski \& Minor, 1997), SHELXT2015 (Sheldrick, 2015a), SHELXL2015 (Sheldrick, 2015b), DIAMOND (Putz \& Brandenburg, 2014) and publCIF (Westrip, 2010).

\section{Acknowledgements}

This work was supported by a Research Grant of Andong National University. The X-ray crystallography experiment at PLS-II BL2D-SMC beamline was supported in part by MSIP and POSTECH.

\section{References}

Choi, J.-H. (2009). Inorg. Chim. Acta, 362, 4231-4236.

Choi, J.-H., Oh, I.-G., Lim, W.-T. \& Park, K.-M. (2004a). Acta Cryst. C60, m238-m240.

Choi, J.-H., Oh, I.-G., Suzuki, T. \& Kaizaki, S. (2004b). J. Mol. Struct. 694, 39-44.

Cohen, M. D., Kargacin, B., Klein, C. B. \& Costa, M. (1993). Crit. Rev. Toxicol. 23, 255-281.

Groom, C. R., Bruno, I. J., Lightfoot, M. P. \& Ward, S. C. (2016). Acta Cryst. B72, 171-179.

Harrowfield, J. M., Miyamae, H., Shand, T. M., Skelton, B., Soudi, A. A. \& White, A. H. (1996). Aust. J. Chem. 49, 1051-1066.

Kalidhasan, S., Kumar, A. S. K., Rajesh, V. \& Rajesh, N. (2016). Coord. Chem. Rev. 317, 157-166.

Kim, N.-H., Hwang, I.-C. \& Ha, K. (2009). Acta Cryst. E65, o2128.

Mireille Ninon, M. O., Fahim, M., Lachkar, M., Marco Contelles, J. L., Perles, J. \& El Bali, B. (2013). Acta Cryst. E69, o1574-o1575.

Moon, D. \& Choi, J.-H. (2016). Acta Cryst. E72, 1417-1420.

Moon, D. \& Choi, J.-H. (2017). Acta Cryst. E73, 403-406. 
Moon, D., Choi, J.-H., Ryoo, K. S. \& Hong, Y. P. (2013). Acta Cryst. E69, m376-m377.

Moon, D., Takase, M., Akitsu, T. \& Choi, J.-H. (2017). Acta Cryst. E73, 72-75.

Moon, D., Tanaka, S., Akitsu, T. \& Choi, J.-H. (2015). Acta Cryst. E71, 1336-1339.

Nave, C. \& Truter, M. R. (1974). J. Chem. Soc. Dalton Trans. pp. 2351-2354.

Otwinowski, Z. \& Minor, W. (1997). Methods in Enzymology, Vol. 276, Macromolecular Crystallography, Part A, edited by C. W. Carter Jr \& R. M. Sweet, pp 307-326. New York: Academic Press. Putz, H. \& Brandenburg, K. (2014). DIAMOND. Crystal Impact GbR, Bonn, Germany.

Robinson, G. H., Sangokoya, S. A., Pennington, W. T., Self, M. F. \& Rogers, R. D. (1989). J. Coord. Chem. 19, 287-294.
Said, S., Mhadhbi, N., Hajlaoui, F., Bataille, T. \& Naïli, H. (2013). Acta Cryst. E69, o1278.

Sheldrick, G. M. (2015a). Acta Cryst. A71, 3-8.

Sheldrick, G. M. (2015b). Acta Cryst. C71, 3-8.

Shin, J. W., Eom, K. \& Moon, D. (2016). J. Synchrotron Rad. 23, 369373.

Subhan, M. A., Choi, J.-H. \& Ng, S. W. (2011). Z. Anorg. Allg. Chem. 637, 2193-2197.

Subramanian, S. \& Zaworotko, M. J. (1995). Can. J. Chem. 73, 414424.

Trabelsi, S., Roisnel, T. \& Marouani, H. (2015). J. Advan. Chem, 11, 3394-3403.

Westrip, S. P. (2010). J. Appl. Cryst. 43, 920-925.

Zhu, R.-Q. (2012). Acta Cryst. E68, m389. 


\section{supporting information}

Acta Cryst. (2017). E73, 755-758 [https://doi.org/10.1107/S2056989017005771]

\section{Crystal structure of 1,4,8,11-tetraazoniacyclotetradecane bis(dichromate) monohydrate from synchrotron data}

\section{Dohyun Moon and Jong-Ha Choi}

\section{Computing details}

Data collection: PAL BL2D-SMDC (Shin et al., 2016); cell refinement: HKL3000sm (Otwinowski \& Minor, 1997); data reduction: HKL3000sm (Otwinowski \& Minor, 1997); program(s) used to solve structure: SHELXT2015 (Sheldrick, 2015a); program(s) used to refine structure: SHELXL2015 (Sheldrick, 2015b); molecular graphics: DIAMOND (Putz \& Brandenburg, 2014); software used to prepare material for publication: publCIF (Westrip, 2010).

\section{1,4,8,11-Tetraazoniacyclotetradecane bis(dichromate) monohydrate}

\section{Crystal data}

$\left(\mathrm{C}_{10} \mathrm{H}_{28} \mathrm{~N}_{4}\right)\left[\mathrm{Cr}_{2} \mathrm{O}_{7}\right]_{2} \cdot \mathrm{H}_{2} \mathrm{O}$

$M_{r}=654.38$

Monoclinic, $P 2_{1} / c$

$a=10.428(2) \AA$

$b=13.961(2) \AA$

$c=15.490(2) \AA$

$\beta=94.671(3)^{\circ}$

$V=2247.6(6) \AA^{3}$

$Z=4$

\section{Data collection}

ADSC Q210 CCD area detector diffractometer

Radiation source: PLSII 2D bending magnet

$\omega$ scan

Absorption correction: empirical (using intensity measurements)

(HKL3000sm Scalepack; Otwinowski \& Minor, 1997)

$T_{\min }=0.942, T_{\max }=1.000$

Refinement

Refinement on $F^{2}$

Least-squares matrix: full

$R\left[F^{2}>2 \sigma\left(F^{2}\right)\right]=0.038$

$w R\left(F^{2}\right)=0.124$

$S=1.09$

6614 reflections

307 parameters

3 restraints

Hydrogen site location: mixed
$F(000)=1336$

$D_{\mathrm{x}}=1.934 \mathrm{Mg} \mathrm{m}^{-3}$

Synchrotron radiation, $\lambda=0.610 \AA$

Cell parameters from 70448 reflections

$\theta=0.4-33.7^{\circ}$

$\mu=1.28 \mathrm{~mm}^{-1}$

$T=200 \mathrm{~K}$

Block, yellow

$0.11 \times 0.10 \times 0.09 \mathrm{~mm}$

12953 measured reflections

6614 independent reflections

5804 reflections with $I>2 \sigma(I)$

$R_{\text {int }}=0.016$

$\theta_{\max }=25.5^{\circ}, \theta_{\min }=1.7^{\circ}$

$h=-14 \rightarrow 14$

$k=-19 \rightarrow 19$

$l=-21 \rightarrow 21$

$\mathrm{H}$ atoms treated by a mixture of independent

and constrained refinement

$w=1 /\left[\sigma^{2}\left(F_{\mathrm{o}}^{2}\right)+(0.0712 P)^{2}+4.2149 P\right]$

where $P=\left(F_{\mathrm{o}}^{2}+2 F_{\mathrm{c}}^{2}\right) / 3$

$(\Delta / \sigma)_{\max }=0.001$

$\Delta \rho_{\max }=2.21 \mathrm{e} \AA^{-3}$

$\Delta \rho_{\min }=-1.04 \mathrm{e} \AA^{-3}$ 
Extinction correction: SHELXL2016 (Sheldrick, 2015b),

$\mathrm{Fc}^{*}=\mathrm{kFc}\left[1+0.001 \mathrm{xFc}^{2} \lambda^{3} / \sin (2 \theta)\right]^{-1 / 4}$

Extinction coefficient: $0.0096(10)$

\section{Special details}

Geometry. All esds (except the esd in the dihedral angle between two 1.s. planes) are estimated using the full covariance matrix. The cell esds are taken into account individually in the estimation of esds in distances, angles and torsion angles; correlations between esds in cell parameters are only used when they are defined by crystal symmetry. An approximate (isotropic) treatment of cell esds is used for estimating esds involving l.s. planes.

Fractional atomic coordinates and isotropic or equivalent isotropic displacement parameters $\left(\AA^{2}\right)$

\begin{tabular}{|c|c|c|c|c|}
\hline & $x$ & $y$ & $z$ & $U_{\text {iso }} * / U_{\text {eq }}$ \\
\hline Cr1A & $0.94583(4)$ & $0.25171(3)$ & $0.36481(2)$ & $0.00893(10)$ \\
\hline $\mathrm{Cr} 2 \mathrm{~A}$ & $0.97052(4)$ & $0.12932(3)$ & $0.18519(2)$ & $0.01054(10)$ \\
\hline O1A & $0.94853(19)$ & $0.23337(12)$ & $0.25072(11)$ & $0.0152(3)$ \\
\hline $\mathrm{O} 2 \mathrm{~A}$ & $0.9464(2)$ & $0.16272(14)$ & $0.08541(12)$ & $0.0215(4)$ \\
\hline $\mathrm{O} 3 \mathrm{~A}$ & $0.8720(2)$ & $0.04430(14)$ & $0.20636(13)$ & $0.0198(4)$ \\
\hline O4A & $1.1160(2)$ & $0.09255(16)$ & $0.20477(14)$ & $0.0239(4)$ \\
\hline $\mathrm{O} 5 \mathrm{~A}$ & $1.05842(18)$ & $0.18684(14)$ & $0.41408(13)$ & $0.0198(4)$ \\
\hline O6A & $0.80867(18)$ & $0.22499(15)$ & $0.39940(13)$ & 0.0207 (4) \\
\hline O7A & $0.9714(2)$ & $0.36520(13)$ & $0.37925(12)$ & 0.0189 (4) \\
\hline Cr1B & $0.49741(4)$ & $0.58036(3)$ & $0.18882(2)$ & $0.01299(10)$ \\
\hline $\mathrm{Cr} 2 \mathrm{~B}$ & $0.45749(4)$ & $0.72591(3)$ & $0.35345(2)$ & $0.01274(10)$ \\
\hline O1B & $0.5392(2)$ & $0.66545(16)$ & $0.27369(13)$ & $0.0226(4)$ \\
\hline $\mathrm{O} 2 \mathrm{~B}$ & $0.3859(2)$ & $0.51137(17)$ & $0.21811(15)$ & $0.0283(5)$ \\
\hline $\mathrm{O} 3 \mathrm{~B}$ & $0.6245(2)$ & $0.51689(16)$ & $0.17659(13)$ & $0.0244(4)$ \\
\hline $\mathrm{O} 4 \mathrm{~B}$ & $0.4512(2)$ & $0.63565(15)$ & $0.09915(13)$ & $0.0225(4)$ \\
\hline O5B & $0.5562(3)$ & $0.8034(2)$ & $0.39672(19)$ & $0.0485(8)$ \\
\hline O6B & $0.4180(2)$ & $0.65172(15)$ & $0.42912(13)$ & $0.0222(4)$ \\
\hline O7B & $0.3338(3)$ & $0.7803(2)$ & $0.31136(17)$ & $0.0442(7)$ \\
\hline N1C & $1.1608(2)$ & $0.48518(17)$ & $0.07746(16)$ & $0.0207(4)$ \\
\hline $\mathrm{H} 1 \mathrm{NC}$ & 1.192696 & 0.527486 & 0.118455 & $0.025^{*}$ \\
\hline $\mathrm{H} 2 \mathrm{NC}$ & 1.084404 & 0.508441 & 0.053719 & $0.025^{*}$ \\
\hline $\mathrm{N} 2 \mathrm{C}$ & $0.8491(2)$ & 0.38005 (16) & $0.07391(15)$ & $0.0179(4)$ \\
\hline $\mathrm{H} 3 \mathrm{NC}$ & 0.905802 & 0.373982 & 0.032598 & $0.021^{*}$ \\
\hline $\mathrm{H} 4 \mathrm{NC}$ & 0.829751 & 0.320363 & 0.092561 & $0.021^{*}$ \\
\hline $\mathrm{C} 1 \mathrm{C}$ & $1.2545(2)$ & $0.47649(17)$ & $0.00725(15)$ & $0.0133(4)$ \\
\hline $\mathrm{H} 1 \mathrm{C} 1$ & 1.338852 & 0.453735 & 0.033274 & $0.016^{*}$ \\
\hline $\mathrm{H} 1 \mathrm{C} 2$ & 1.221515 & 0.428903 & -0.036475 & $0.016^{*}$ \\
\hline $\mathrm{C} 2 \mathrm{C}$ & $1.1387(2)$ & 0.38925 (17) & $0.11984(15)$ & $0.0129(4)$ \\
\hline $\mathrm{H} 2 \mathrm{C} 1$ & 1.110865 & 0.341470 & 0.074936 & $0.016^{*}$ \\
\hline $\mathrm{H} 2 \mathrm{C} 2$ & 1.219961 & 0.366488 & 0.150578 & $0.016^{*}$ \\
\hline $\mathrm{C} 3 \mathrm{C}$ & $1.0374(2)$ & $0.39918(15)$ & $0.18280(12)$ & $0.0071(3)$ \\
\hline $\mathrm{H} 3 \mathrm{C} 1$ & 1.022978 & 0.335164 & 0.207745 & $0.008 *$ \\
\hline $\mathrm{H} 3 \mathrm{C} 2$ & 1.072279 & 0.440867 & 0.230818 & $0.008^{*}$ \\
\hline $\mathrm{C} 4 \mathrm{C}$ & $0.9100(2)$ & $0.43839(17)$ & $0.14937(15)$ & $0.0125(4)$ \\
\hline $\mathrm{H} 4 \mathrm{C} 1$ & 0.920930 & 0.505402 & 0.130394 & $0.015^{*}$ \\
\hline
\end{tabular}




$\begin{array}{lllll}\mathrm{H} 4 \mathrm{C} 2 & 0.851635 & 0.438789 & 0.196698 & 0.015^{*} \\ \mathrm{C} 5 \mathrm{C} & 0.72907(19) & 0.42821(15) & 0.03596(13) & 0.0068(3) \\ \mathrm{H} 5 \mathrm{C} 1 & 0.671037 & 0.437307 & 0.082792 & 0.008^{*} \\ \mathrm{H} 5 \mathrm{C} 2 & 0.685296 & 0.384273 & -0.007113 & 0.008^{*} \\ \mathrm{~N} 1 \mathrm{D} & 0.74042(19) & 0.43291(15) & 0.45783(13) & 0.0124(4) \\ \mathrm{H} 1 \mathrm{ND} & 0.797863 & 0.387321 & 0.444322 & 0.015^{*} \\ \mathrm{H} 2 \mathrm{ND} & 0.694753 & 0.408892 & 0.500537 & 0.015^{*} \\ \mathrm{~N} 2 \mathrm{D} & 0.6336(2) & 0.57400(17) & 0.58080(16) & 0.0213(5) \\ \mathrm{H} 3 \mathrm{ND} & 0.589300 & 0.521117 & 0.561168 & 0.026^{*} \\ \mathrm{H} 4 \mathrm{ND} & 0.676852 & 0.559214 & 0.632478 & 0.026^{*} \\ \mathrm{C} 1 \mathrm{D} & 0.5652(2) & 0.36748(17) & 0.35112(15) & 0.0139(4) \\ \mathrm{H} 1 \mathrm{D} 1 & 0.527317 & 0.380206 & 0.291539 & 0.017^{*} \\ \mathrm{H} 1 \mathrm{D} 2 & 0.619964 & 0.309743 & 0.348804 & 0.017^{*} \\ \mathrm{C} 2 \mathrm{D} & 0.6505(2) & 0.45172(18) & 0.38007(15) & 0.0131(4) \\ \mathrm{H} 2 \mathrm{D} 1 & 0.701386 & 0.470585 & 0.331598 & 0.016^{*} \\ \mathrm{H} 2 \mathrm{D} 2 & 0.594811 & 0.506624 & 0.392587 & 0.016^{*} \\ \mathrm{C} 3 \mathrm{D} & 0.8139(2) & 0.52031(18) & 0.49225(16) & 0.0144(4) \\ \mathrm{H} 3 \mathrm{D} 1 & 0.870329 & 0.542564 & 0.447912 & 0.017^{*} \\ \mathrm{H} 3 \mathrm{D} 2 & 0.869859 & 0.501556 & 0.544205 & 0.017^{*} \\ \mathrm{C} 4 \mathrm{D} & 0.7290(2) & 0.60301(18) & 0.51601(16) & 0.0142(4) \\ \mathrm{H} 4 \mathrm{D} 1 & 0.680965 & 0.627588 & 0.462816 & 0.017^{*} \\ \mathrm{H} 4 \mathrm{D} 2 & 0.783948 & 0.655524 & 0.541016 & 0.017^{*} \\ \text { C5D } & 0.5418(2) & 0.65411(15) & 0.59291(14) & 0.0084(4) \\ \text { H5D1 } & 0.503368 & 0.674117 & 0.535164 & 0.010^{*} \\ \text { H5D2 } & 0.591171 & 0.709254 & 0.618333 & 0.010^{*} \\ \text { O1W } & 0.7376(2) & 0.75592(18) & 0.19670(18) & 0.0313(5) \\ \text { H1O1 } & 0.749(13) & 0.723(5) & 0.152(4) & 0.30(8)^{*} \\ \text { H2O1 } & 0.708(5) & 0.718(3) & 0.232(3) & 0.066(17)^{*} \\ & & & & \end{array}$

Atomic displacement parameters $\left(\AA^{2}\right)$

\begin{tabular}{lllllll}
\hline & $U^{11}$ & $U^{22}$ & $U^{33}$ & $U^{12}$ & $U^{13}$ & $U^{23}$ \\
\hline Cr1A & $0.01239(17)$ & $0.00745(17)$ & $0.00736(17)$ & $0.00232(12)$ & $0.00323(12)$ & $0.00134(12)$ \\
Cr2A & $0.01754(19)$ & $0.00764(17)$ & $0.00646(17)$ & $0.00091(13)$ & $0.00113(12)$ & $-0.00038(12)$ \\
O1A & $0.0271(9)$ & $0.0096(7)$ & $0.0094(7)$ & $0.0002(7)$ & $0.0048(6)$ & $-0.0016(6)$ \\
O2A & $0.0375(11)$ & $0.0171(9)$ & $0.0098(8)$ & $0.0022(8)$ & $0.0012(7)$ & $0.0016(7)$ \\
O3A & $0.0267(10)$ & $0.0128(8)$ & $0.0197(9)$ & $-0.0043(7)$ & $-0.0002(7)$ & $0.0030(7)$ \\
O4A & $0.0219(9)$ & $0.0260(10)$ & $0.0238(10)$ & $0.0072(8)$ & $0.0012(8)$ & $-0.0015(8)$ \\
O5A & $0.0186(9)$ & $0.0214(9)$ & $0.0194(9)$ & $0.0085(7)$ & $0.0013(7)$ & $0.0053(7)$ \\
O6A & $0.0161(8)$ & $0.0260(10)$ & $0.0209(9)$ & $0.0013(7)$ & $0.0059(7)$ & $0.0078(8)$ \\
O7A & $0.0306(10)$ & $0.0104(8)$ & $0.0159(8)$ & $0.0000(7)$ & $0.0036(7)$ & $-0.0028(6)$ \\
Cr1B & $0.01447(19)$ & $0.01425(19)$ & $0.01028(18)$ & $0.00271(13)$ & $0.00115(13)$ & $-0.00104(13)$ \\
Cr2B & $0.0189(2)$ & $0.01113(18)$ & $0.00895(17)$ & $0.00155(13)$ & $0.00592(13)$ & $0.00160(13)$ \\
O1B & $0.0241(9)$ & $0.0264(10)$ & $0.0181(9)$ & $-0.0004(8)$ & $0.0067(7)$ & $-0.0098(8)$ \\
O2B & $0.0254(10)$ & $0.0306(11)$ & $0.0292(11)$ & $-0.0093(9)$ & $0.0038(8)$ & $0.0042(9)$ \\
O3B & $0.0250(10)$ & $0.0290(11)$ & $0.0194(9)$ & $0.0134(8)$ & $0.0023(7)$ & $-0.0046(8)$ \\
O4B & $0.0276(10)$ & $0.0218(10)$ & $0.0171(9)$ & $0.0010(8)$ & $-0.0051(7)$ & $0.0036(7)$ \\
O5B & $0.0656(19)$ & $0.0379(14)$ & $0.0446(15)$ & $-0.0317(14)$ & $0.0206(14)$ & $-0.0224(12)$ \\
& & & & & &
\end{tabular}




\begin{tabular}{lllllll} 
& & & & \\
O6B & $0.0291(10)$ & $0.0231(10)$ & $0.0149(8)$ & $-0.0037(8)$ & $0.0040(7)$ & $0.0079(7)$ \\
O7B & $0.0396(14)$ & $0.0612(18)$ & $0.0347(13)$ & $0.0314(13)$ & $0.0199(11)$ & $0.0275(13)$ \\
N1C & $0.0239(11)$ & $0.0177(10)$ & $0.0207(10)$ & $0.0008(9)$ & $0.0035(8)$ & $0.0042(9)$ \\
N2C & $0.0230(11)$ & $0.0143(10)$ & $0.0165(10)$ & $-0.0009(8)$ & $0.0028(8)$ & $-0.0007(8)$ \\
C1C & $0.0150(10)$ & $0.0110(10)$ & $0.0140(10)$ & $0.0001(8)$ & $0.0020(8)$ & $0.0013(8)$ \\
C2C & $0.0151(10)$ & $0.0113(10)$ & $0.0125(9)$ & $0.0006(8)$ & $0.0011(8)$ & $0.0028(8)$ \\
C3C & $0.0131(9)$ & $0.0052(8)$ & $0.0028(8)$ & $-0.0017(7)$ & $0.0005(7)$ & $0.0022(6)$ \\
C4C & $0.0161(10)$ & $0.0119(10)$ & $0.0098(9)$ & $0.0001(8)$ & $0.0019(8)$ & $-0.0017(8)$ \\
C5C & $0.0083(8)$ & $0.0076(8)$ & $0.0046(8)$ & $-0.0029(7)$ & $0.0020(6)$ & $0.0005(7)$ \\
N1D & $0.0140(9)$ & $0.0138(9)$ & $0.0096(8)$ & $0.0049(7)$ & $0.0021(7)$ & $0.0015(7)$ \\
N2D & $0.0226(11)$ & $0.0189(11)$ & $0.0228(11)$ & $0.0038(9)$ & $0.0039(9)$ & $0.0000(9)$ \\
C1D & $0.0164(10)$ & $0.0149(10)$ & $0.0106(10)$ & $0.0037(8)$ & $0.0017(8)$ & $-0.0030(8)$ \\
C2D & $0.0136(10)$ & $0.0159(10)$ & $0.0098(9)$ & $0.0021(8)$ & $0.0000(7)$ & $0.0024(8)$ \\
C3D & $0.0108(9)$ & $0.0183(11)$ & $0.0141(10)$ & $0.0000(8)$ & $0.0021(8)$ & $0.0024(9)$ \\
C4D & $0.0148(10)$ & $0.0128(10)$ & $0.0152(10)$ & $-0.0017(8)$ & $0.0019(8)$ & $-0.0002(8)$ \\
C5D & $0.0107(9)$ & $0.0054(8)$ & $0.0093(9)$ & $0.0017(7)$ & $0.0018(7)$ & $-0.0019(7)$ \\
O1W & $0.0229(10)$ & $0.0311(12)$ & $0.0405(13)$ & $0.0049(9)$ & $0.0053(9)$ & $0.0165(10)$ \\
& & & & & & \\
\hline
\end{tabular}

Geometric parameters $\left(\AA,{ }^{\circ}\right)$

\begin{tabular}{|c|c|c|c|}
\hline $\mathrm{Cr} 1 \mathrm{~A}-\mathrm{O} 6 \mathrm{~A}$ & 1.6114 (19) & $\mathrm{C} 3 \mathrm{C}-\mathrm{C} 4 \mathrm{C}$ & $1.491(3)$ \\
\hline $\mathrm{Cr} 1 \mathrm{~A}-\mathrm{O} 7 \mathrm{~A}$ & $1.6192(19)$ & $\mathrm{C} 3 \mathrm{C}-\mathrm{H} 3 \mathrm{C} 1$ & 0.9900 \\
\hline $\mathrm{Cr} 1 \mathrm{~A}-\mathrm{O} 5 \mathrm{~A}$ & $1.6233(19)$ & $\mathrm{C} 3 \mathrm{C}-\mathrm{H} 3 \mathrm{C} 2$ & 0.9900 \\
\hline $\mathrm{Cr} 1 \mathrm{~A}-\mathrm{O} 1 \mathrm{~A}$ & $1.7882(18)$ & $\mathrm{C} 4 \mathrm{C}-\mathrm{H} 4 \mathrm{C} 1$ & 0.9900 \\
\hline $\mathrm{Cr} 2 \mathrm{~A}-\mathrm{O} 4 \mathrm{~A}$ & $1.607(2)$ & $\mathrm{C} 4 \mathrm{C}-\mathrm{H} 4 \mathrm{C} 2$ & 0.9900 \\
\hline $\mathrm{Cr} 2 \mathrm{~A}-\mathrm{O} 2 \mathrm{~A}$ & $1.6143(19)$ & $\mathrm{C} 5 \mathrm{C}-\mathrm{H} 5 \mathrm{C} 1$ & 0.9900 \\
\hline $\mathrm{Cr} 2 \mathrm{~A}-\mathrm{O} 3 \mathrm{~A}$ & 1.6209 (19) & $\mathrm{C} 5 \mathrm{C}-\mathrm{H} 5 \mathrm{C} 2$ & 0.9900 \\
\hline $\mathrm{Cr} 2 \mathrm{~A}-\mathrm{O} 1 \mathrm{~A}$ & $1.7975(18)$ & $\mathrm{N} 1 \mathrm{D}-\mathrm{C} 2 \mathrm{D}$ & $1.489(3)$ \\
\hline $\mathrm{Cr} 1 \mathrm{~B}-\mathrm{O} 2 \mathrm{~B}$ & $1.603(2)$ & $\mathrm{N} 1 \mathrm{D}-\mathrm{C} 3 \mathrm{D}$ & $1.515(3)$ \\
\hline $\mathrm{Cr} 1 \mathrm{~B}-\mathrm{O} 3 \mathrm{~B}$ & $1.618(2)$ & N1D-H1ND & 0.9100 \\
\hline $\mathrm{Cr} 1 \mathrm{~B}-\mathrm{O} 4 \mathrm{~B}$ & $1.627(2)$ & $\mathrm{N} 1 \mathrm{D}-\mathrm{H} 2 \mathrm{ND}$ & 0.9100 \\
\hline $\mathrm{Cr} 1 \mathrm{~B}-\mathrm{O} 1 \mathrm{~B}$ & $1.799(2)$ & $\mathrm{N} 2 \mathrm{D}-\mathrm{C} 5 \mathrm{D}$ & $1.494(3)$ \\
\hline $\mathrm{Cr} 2 \mathrm{~B}-\mathrm{O} 7 \mathrm{~B}$ & $1.590(2)$ & $\mathrm{N} 2 \mathrm{D}-\mathrm{C} 4 \mathrm{D}$ & $1.524(3)$ \\
\hline $\mathrm{Cr} 2 \mathrm{~B}-\mathrm{O} 5 \mathrm{~B}$ & $1.602(3)$ & $\mathrm{N} 2 \mathrm{D}-\mathrm{H} 3 \mathrm{ND}$ & 0.9100 \\
\hline $\mathrm{Cr} 2 \mathrm{~B}-\mathrm{O} 6 \mathrm{~B}$ & $1.6417(19)$ & $\mathrm{N} 2 \mathrm{D}-\mathrm{H} 4 \mathrm{ND}$ & 0.9100 \\
\hline $\mathrm{Cr} 2 \mathrm{~B}-\mathrm{O} 1 \mathrm{~B}$ & 1.7711 (19) & $\mathrm{C} 1 \mathrm{D}-\mathrm{C} 5 \mathrm{D}^{\mathrm{ii}}$ & $1.498(3)$ \\
\hline $\mathrm{N} 1 \mathrm{C}-\mathrm{C} 2 \mathrm{C}$ & $1.517(3)$ & $\mathrm{C} 1 \mathrm{D}-\mathrm{C} 2 \mathrm{D}$ & $1.520(3)$ \\
\hline $\mathrm{N} 1 \mathrm{C}-\mathrm{C} 1 \mathrm{C}$ & $1.524(3)$ & C1D-H1D1 & 0.9900 \\
\hline $\mathrm{N} 1 \mathrm{C}-\mathrm{H} 1 \mathrm{NC}$ & 0.9100 & C1D-H1D2 & 0.9900 \\
\hline $\mathrm{N} 1 \mathrm{C}-\mathrm{H} 2 \mathrm{NC}$ & 0.9100 & $\mathrm{C} 2 \mathrm{D}-\mathrm{H} 2 \mathrm{D} 1$ & 0.9900 \\
\hline $\mathrm{N} 2 \mathrm{C}-\mathrm{C} 5 \mathrm{C}$ & $1.498(3)$ & $\mathrm{C} 2 \mathrm{D}-\mathrm{H} 2 \mathrm{D} 2$ & 0.9900 \\
\hline $\mathrm{N} 2 \mathrm{C}-\mathrm{C} 4 \mathrm{C}$ & $1.521(3)$ & $\mathrm{C} 3 \mathrm{D}-\mathrm{C} 4 \mathrm{D}$ & $1.519(3)$ \\
\hline $\mathrm{N} 2 \mathrm{C}-\mathrm{H} 3 \mathrm{NC}$ & 0.9100 & $\mathrm{C} 3 \mathrm{D}-\mathrm{H} 3 \mathrm{D} 1$ & 0.9900 \\
\hline $\mathrm{N} 2 \mathrm{C}-\mathrm{H} 4 \mathrm{NC}$ & 0.9100 & $\mathrm{C} 3 \mathrm{D}-\mathrm{H} 3 \mathrm{D} 2$ & 0.9900 \\
\hline $\mathrm{C} 1 \mathrm{C}-\mathrm{C} 5 \mathrm{C}^{\mathrm{i}}$ & $1.506(3)$ & $\mathrm{C} 4 \mathrm{D}-\mathrm{H} 4 \mathrm{D} 1$ & 0.9900 \\
\hline $\mathrm{C} 1 \mathrm{C}-\mathrm{H} 1 \mathrm{C} 1$ & 0.9900 & $\mathrm{C} 4 \mathrm{D}-\mathrm{H} 4 \mathrm{D} 2$ & 0.9900 \\
\hline $\mathrm{C} 1 \mathrm{C}-\mathrm{H} 1 \mathrm{C} 2$ & 0.9900 & $\mathrm{C} 5 \mathrm{D}-\mathrm{H} 5 \mathrm{D} 1$ & 0.9900 \\
\hline $\mathrm{C} 2 \mathrm{C}-\mathrm{C} 3 \mathrm{C}$ & $1.501(3)$ & $\mathrm{C} 5 \mathrm{D}-\mathrm{H} 5 \mathrm{D} 2$ & 0.9900 \\
\hline
\end{tabular}




\begin{tabular}{|c|c|c|c|}
\hline $\mathrm{C} 2 \mathrm{C}-\mathrm{H} 2 \mathrm{C} 1$ & 0.9900 & $\mathrm{O} 1 \mathrm{~W}-\mathrm{H} 1 \mathrm{O} 1$ & $0.844(10)$ \\
\hline $\mathrm{C} 2 \mathrm{C}-\mathrm{H} 2 \mathrm{C} 2$ & 0.9900 & $\mathrm{O} 1 \mathrm{~W}-\mathrm{H} 2 \mathrm{O} 1$ & $0.844(10)$ \\
\hline $\mathrm{O} 6 \mathrm{~A}-\mathrm{Cr} 1 \mathrm{~A}-\mathrm{O} 7 \mathrm{~A}$ & $108.74(11)$ & $\mathrm{C} 3 \mathrm{C}-\mathrm{C} 4 \mathrm{C}-\mathrm{N} 2 \mathrm{C}$ & $112.02(19)$ \\
\hline $\mathrm{O} 6 \mathrm{~A}-\mathrm{Cr} 1 \mathrm{~A}-\mathrm{O} 5 \mathrm{~A}$ & $110.00(10)$ & $\mathrm{C} 3 \mathrm{C}-\mathrm{C} 4 \mathrm{C}-\mathrm{H} 4 \mathrm{C} 1$ & 109.2 \\
\hline $\mathrm{O} 7 \mathrm{~A}-\mathrm{Cr} 1 \mathrm{~A}-\mathrm{O} 5 \mathrm{~A}$ & $112.10(11)$ & $\mathrm{N} 2 \mathrm{C}-\mathrm{C} 4 \mathrm{C}-\mathrm{H} 4 \mathrm{C} 1$ & 109.2 \\
\hline $\mathrm{O} 6 \mathrm{~A}-\mathrm{Cr} 1 \mathrm{~A}-\mathrm{O} 1 \mathrm{~A}$ & $112.41(10)$ & $\mathrm{C} 3 \mathrm{C}-\mathrm{C} 4 \mathrm{C}-\mathrm{H} 4 \mathrm{C} 2$ & 109.2 \\
\hline $\mathrm{O} 7 \mathrm{~A}-\mathrm{Cr} 1 \mathrm{~A}-\mathrm{O} 1 \mathrm{~A}$ & $105.13(9)$ & $\mathrm{N} 2 \mathrm{C}-\mathrm{C} 4 \mathrm{C}-\mathrm{H} 4 \mathrm{C} 2$ & 109.2 \\
\hline $\mathrm{O} 5 \mathrm{~A}-\mathrm{Cr} 1 \mathrm{~A}-\mathrm{O} 1 \mathrm{~A}$ & $108.41(9)$ & $\mathrm{H} 4 \mathrm{C} 1-\mathrm{C} 4 \mathrm{C}-\mathrm{H} 4 \mathrm{C} 2$ & 107.9 \\
\hline $\mathrm{O} 4 \mathrm{~A}-\mathrm{Cr} 2 \mathrm{~A}-\mathrm{O} 2 \mathrm{~A}$ & $110.11(11)$ & $\mathrm{N} 2 \mathrm{C}-\mathrm{C} 5 \mathrm{C}-\mathrm{C} 1 \mathrm{C}^{\mathrm{i}}$ & $116.69(18)$ \\
\hline $\mathrm{O} 4 \mathrm{~A}-\mathrm{Cr} 2 \mathrm{~A}-\mathrm{O} 3 \mathrm{~A}$ & $109.41(11)$ & $\mathrm{N} 2 \mathrm{C}-\mathrm{C} 5 \mathrm{C}-\mathrm{H} 5 \mathrm{C} 1$ & 108.1 \\
\hline $\mathrm{O} 2 \mathrm{~A}-\mathrm{Cr} 2 \mathrm{~A}-\mathrm{O} 3 \mathrm{~A}$ & $110.70(11)$ & $\mathrm{C} 1 \mathrm{C}-\mathrm{C} 5 \mathrm{C}-\mathrm{H} 5 \mathrm{C} 1$ & 108.1 \\
\hline $\mathrm{O} 4 \mathrm{~A}-\mathrm{Cr} 2 \mathrm{~A}-\mathrm{O} 1 \mathrm{~A}$ & $108.27(10)$ & $\mathrm{N} 2 \mathrm{C}-\mathrm{C} 5 \mathrm{C}-\mathrm{H} 5 \mathrm{C} 2$ & 108.1 \\
\hline $\mathrm{O} 2 \mathrm{~A}-\mathrm{Cr} 2 \mathrm{~A}-\mathrm{O} 1 \mathrm{~A}$ & $106.87(9)$ & $\mathrm{C} 1 \mathrm{C}-\mathrm{C} 5 \mathrm{C}-\mathrm{H} 5 \mathrm{C} 2$ & 108.1 \\
\hline $\mathrm{O} 3 \mathrm{~A}-\mathrm{Cr} 2 \mathrm{~A}-\mathrm{O} 1 \mathrm{~A}$ & $111.43(10)$ & $\mathrm{H} 5 \mathrm{C} 1-\mathrm{C} 5 \mathrm{C}-\mathrm{H} 5 \mathrm{C} 2$ & 107.3 \\
\hline $\mathrm{Cr} 1 \mathrm{~A}-\mathrm{O} 1 \mathrm{~A}-\mathrm{Cr} 2 \mathrm{~A}$ & $133.37(11)$ & $\mathrm{C} 2 \mathrm{D}-\mathrm{N} 1 \mathrm{D}-\mathrm{C} 3 \mathrm{D}$ & $114.21(19)$ \\
\hline $\mathrm{O} 2 \mathrm{~B}-\mathrm{Cr} 1 \mathrm{~B}-\mathrm{O} 3 \mathrm{~B}$ & $108.89(13)$ & $\mathrm{C} 2 \mathrm{D}-\mathrm{N} 1 \mathrm{D}-\mathrm{H} 1 \mathrm{ND}$ & 108.7 \\
\hline $\mathrm{O} 2 \mathrm{~B}-\mathrm{Cr} 1 \mathrm{~B}-\mathrm{O} 4 \mathrm{~B}$ & $110.81(11)$ & $\mathrm{C} 3 \mathrm{D}-\mathrm{N} 1 \mathrm{D}-\mathrm{H} 1 \mathrm{ND}$ & 108.7 \\
\hline $\mathrm{O} 3 \mathrm{~B}-\mathrm{Cr} 1 \mathrm{~B}-\mathrm{O} 4 \mathrm{~B}$ & $110.38(11)$ & $\mathrm{C} 2 \mathrm{D}-\mathrm{N} 1 \mathrm{D}-\mathrm{H} 2 \mathrm{ND}$ & 108.7 \\
\hline $\mathrm{O} 2 \mathrm{~B}-\mathrm{Cr} 1 \mathrm{~B}-\mathrm{O} 1 \mathrm{~B}$ & $109.15(11)$ & $\mathrm{C} 3 \mathrm{D}-\mathrm{N} 1 \mathrm{D}-\mathrm{H} 2 \mathrm{ND}$ & 108.7 \\
\hline $\mathrm{O} 3 \mathrm{~B}-\mathrm{Cr} 1 \mathrm{~B}-\mathrm{O} 1 \mathrm{~B}$ & $107.16(10)$ & $\mathrm{H} 1 \mathrm{ND}-\mathrm{N} 1 \mathrm{D}-\mathrm{H} 2 \mathrm{ND}$ & 107.6 \\
\hline $\mathrm{O} 4 \mathrm{~B}-\mathrm{Cr} 1 \mathrm{~B}-\mathrm{O} 1 \mathrm{~B}$ & $110.35(11)$ & $\mathrm{C} 5 \mathrm{D}-\mathrm{N} 2 \mathrm{D}-\mathrm{C} 4 \mathrm{D}$ & $110.1(2)$ \\
\hline $\mathrm{O} 7 \mathrm{~B}-\mathrm{Cr} 2 \mathrm{~B}-\mathrm{O} 5 \mathrm{~B}$ & $108.74(19)$ & $\mathrm{C} 5 \mathrm{D}-\mathrm{N} 2 \mathrm{D}-\mathrm{H} 3 \mathrm{ND}$ & 109.6 \\
\hline $\mathrm{O} 7 \mathrm{~B}-\mathrm{Cr} 2 \mathrm{~B}-\mathrm{O} 6 \mathrm{~B}$ & $110.59(12)$ & $\mathrm{C} 4 \mathrm{D}-\mathrm{N} 2 \mathrm{D}-\mathrm{H} 3 \mathrm{ND}$ & 109.6 \\
\hline $\mathrm{O} 5 \mathrm{~B}-\mathrm{Cr} 2 \mathrm{~B}-\mathrm{O} 6 \mathrm{~B}$ & $108.50(14)$ & $\mathrm{C} 5 \mathrm{D}-\mathrm{N} 2 \mathrm{D}-\mathrm{H} 4 \mathrm{ND}$ & 109.6 \\
\hline $\mathrm{O} 7 \mathrm{~B}-\mathrm{Cr} 2 \mathrm{~B}-\mathrm{O} 1 \mathrm{~B}$ & $111.22(12)$ & $\mathrm{C} 4 \mathrm{D}-\mathrm{N} 2 \mathrm{D}-\mathrm{H} 4 \mathrm{ND}$ & 109.6 \\
\hline $\mathrm{O} 5 \mathrm{~B}-\mathrm{Cr} 2 \mathrm{~B}-\mathrm{O} 1 \mathrm{~B}$ & $106.51(13)$ & $\mathrm{H} 3 \mathrm{ND}-\mathrm{N} 2 \mathrm{D}-\mathrm{H} 4 \mathrm{ND}$ & 108.2 \\
\hline $\mathrm{O} 6 \mathrm{~B}-\mathrm{Cr} 2 \mathrm{~B}-\mathrm{O} 1 \mathrm{~B}$ & $111.12(11)$ & $\mathrm{C} 5 \mathrm{D}^{\mathrm{ii}}-\mathrm{C} 1 \mathrm{D}-\mathrm{C} 2 \mathrm{D}$ & $115.47(19)$ \\
\hline $\mathrm{Cr} 2 \mathrm{~B}-\mathrm{O} 1 \mathrm{~B}-\mathrm{Cr} 1 \mathrm{~B}$ & $136.28(12)$ & $\mathrm{C} 5 \mathrm{D}^{\mathrm{ii}}-\mathrm{C} 1 \mathrm{D}-\mathrm{H} 1 \mathrm{D} 1$ & 108.4 \\
\hline $\mathrm{C} 2 \mathrm{C}-\mathrm{N} 1 \mathrm{C}-\mathrm{C} 1 \mathrm{C}$ & $111.5(2)$ & $\mathrm{C} 2 \mathrm{D}-\mathrm{C} 1 \mathrm{D}-\mathrm{H} 1 \mathrm{D} 1$ & 108.4 \\
\hline $\mathrm{C} 2 \mathrm{C}-\mathrm{N} 1 \mathrm{C}-\mathrm{H} 1 \mathrm{NC}$ & 109.3 & $\mathrm{C} 5 \mathrm{D}^{\mathrm{ii}}-\mathrm{C} 1 \mathrm{D}-\mathrm{H} 1 \mathrm{D} 2$ & 108.4 \\
\hline $\mathrm{C} 1 \mathrm{C}-\mathrm{N} 1 \mathrm{C}-\mathrm{H} 1 \mathrm{NC}$ & 109.3 & $\mathrm{C} 2 \mathrm{D}-\mathrm{C} 1 \mathrm{D}-\mathrm{H} 1 \mathrm{D} 2$ & 108.4 \\
\hline $\mathrm{C} 2 \mathrm{C}-\mathrm{N} 1 \mathrm{C}-\mathrm{H} 2 \mathrm{NC}$ & 109.3 & H1D1-C1D-H1D2 & 107.5 \\
\hline $\mathrm{C} 1 \mathrm{C}-\mathrm{N} 1 \mathrm{C}-\mathrm{H} 2 \mathrm{NC}$ & 109.3 & $\mathrm{~N} 1 \mathrm{D}-\mathrm{C} 2 \mathrm{D}-\mathrm{C} 1 \mathrm{D}$ & $114.7(2)$ \\
\hline $\mathrm{H} 1 \mathrm{NC}-\mathrm{N} 1 \mathrm{C}-\mathrm{H} 2 \mathrm{NC}$ & 108.0 & $\mathrm{~N} 1 \mathrm{D}-\mathrm{C} 2 \mathrm{D}-\mathrm{H} 2 \mathrm{D} 1$ & 108.6 \\
\hline $\mathrm{C} 5 \mathrm{C}-\mathrm{N} 2 \mathrm{C}-\mathrm{C} 4 \mathrm{C}$ & $110.15(18)$ & $\mathrm{C} 1 \mathrm{D}-\mathrm{C} 2 \mathrm{D}-\mathrm{H} 2 \mathrm{D} 1$ & 108.6 \\
\hline $\mathrm{C} 5 \mathrm{C}-\mathrm{N} 2 \mathrm{C}-\mathrm{H} 3 \mathrm{NC}$ & 109.6 & $\mathrm{~N} 1 \mathrm{D}-\mathrm{C} 2 \mathrm{D}-\mathrm{H} 2 \mathrm{D} 2$ & 108.6 \\
\hline $\mathrm{C} 4 \mathrm{C}-\mathrm{N} 2 \mathrm{C}-\mathrm{H} 3 \mathrm{NC}$ & 109.6 & $\mathrm{C} 1 \mathrm{D}-\mathrm{C} 2 \mathrm{D}-\mathrm{H} 2 \mathrm{D} 2$ & 108.6 \\
\hline $\mathrm{C} 5 \mathrm{C}-\mathrm{N} 2 \mathrm{C}-\mathrm{H} 4 \mathrm{NC}$ & 109.6 & $\mathrm{H} 2 \mathrm{D} 1-\mathrm{C} 2 \mathrm{D}-\mathrm{H} 2 \mathrm{D} 2$ & 107.6 \\
\hline $\mathrm{C} 4 \mathrm{C}-\mathrm{N} 2 \mathrm{C}-\mathrm{H} 4 \mathrm{NC}$ & 109.6 & $\mathrm{~N} 1 \mathrm{D}-\mathrm{C} 3 \mathrm{D}-\mathrm{C} 4 \mathrm{D}$ & $114.17(19)$ \\
\hline $\mathrm{H} 3 \mathrm{NC}-\mathrm{N} 2 \mathrm{C}-\mathrm{H} 4 \mathrm{NC}$ & 108.1 & N1D-C3D-H3D1 & 108.7 \\
\hline $\mathrm{C} 5 \mathrm{C}^{\mathrm{i}}-\mathrm{C} 1 \mathrm{C}-\mathrm{N} 1 \mathrm{C}$ & $110.47(19)$ & $\mathrm{C} 4 \mathrm{D}-\mathrm{C} 3 \mathrm{D}-\mathrm{H} 3 \mathrm{D} 1$ & 108.7 \\
\hline $\mathrm{C} 5 \mathrm{C}^{\mathrm{i}}-\mathrm{C} 1 \mathrm{C}-\mathrm{H} 1 \mathrm{C} 1$ & 109.6 & $\mathrm{~N} 1 \mathrm{D}-\mathrm{C} 3 \mathrm{D}-\mathrm{H} 3 \mathrm{D} 2$ & 108.7 \\
\hline $\mathrm{N} 1 \mathrm{C}-\mathrm{C} 1 \mathrm{C}-\mathrm{H} 1 \mathrm{C} 1$ & 109.6 & $\mathrm{C} 4 \mathrm{D}-\mathrm{C} 3 \mathrm{D}-\mathrm{H} 3 \mathrm{D} 2$ & 108.7 \\
\hline $\mathrm{C} 5 \mathrm{C}^{\mathrm{i}}-\mathrm{C} 1 \mathrm{C}-\mathrm{H} 1 \mathrm{C} 2$ & 109.6 & $\mathrm{H} 3 \mathrm{D} 1-\mathrm{C} 3 \mathrm{D}-\mathrm{H} 3 \mathrm{D} 2$ & 107.6 \\
\hline $\mathrm{N} 1 \mathrm{C}-\mathrm{C} 1 \mathrm{C}-\mathrm{H} 1 \mathrm{C} 2$ & 109.6 & $\mathrm{C} 3 \mathrm{D}-\mathrm{C} 4 \mathrm{D}-\mathrm{N} 2 \mathrm{D}$ & $112.5(2)$ \\
\hline $\mathrm{H} 1 \mathrm{C} 1-\mathrm{C} 1 \mathrm{C}-\mathrm{H} 1 \mathrm{C} 2$ & 108.1 & $\mathrm{C} 3 \mathrm{D}-\mathrm{C} 4 \mathrm{D}-\mathrm{H} 4 \mathrm{D} 1$ & 109.1 \\
\hline $\mathrm{C} 3 \mathrm{C}-\mathrm{C} 2 \mathrm{C}-\mathrm{N} 1 \mathrm{C}$ & $109.84(19)$ & $\mathrm{N} 2 \mathrm{D}-\mathrm{C} 4 \mathrm{D}-\mathrm{H} 4 \mathrm{D} 1$ & 109.1 \\
\hline
\end{tabular}




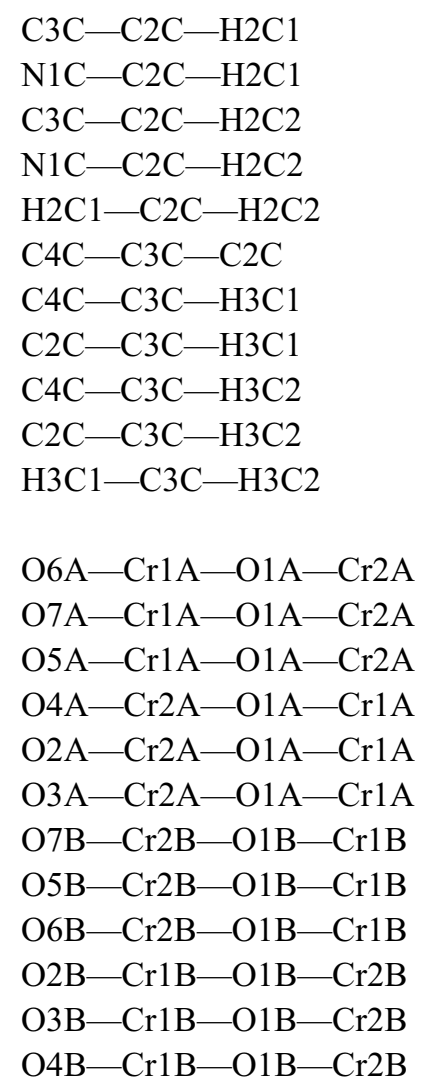

109.7

109.7

109.7

109.7

108.2

$117.56(18)$

107.9

107.9

107.9

107.9

107.2

$-81.13(17)$

$160.74(15)$

$40.70(18)$

$-67.38(18)$

$174.04(15)$

$52.99(18)$

$-53.2(2)$

$-171.5(2)$

$70.5(2)$

$-32.7(2)$

$-150.48(18)$

$89.3(2)$
$\mathrm{C} 3 \mathrm{D}-\mathrm{C} 4 \mathrm{D}-\mathrm{H} 4 \mathrm{D} 2$

$\mathrm{N} 2 \mathrm{D}-\mathrm{C} 4 \mathrm{D}-\mathrm{H} 4 \mathrm{D} 2$

$\mathrm{H} 4 \mathrm{D} 1-\mathrm{C} 4 \mathrm{D}-\mathrm{H} 4 \mathrm{D} 2$

$\mathrm{N} 2 \mathrm{D}-\mathrm{C} 5 \mathrm{D}-\mathrm{C} 1 \mathrm{D}^{\mathrm{ii}}$

$\mathrm{N} 2 \mathrm{D}-\mathrm{C} 5 \mathrm{D}-\mathrm{H} 5 \mathrm{D} 1$

C1D ${ }^{\mathrm{ii}}-\mathrm{C} 5 \mathrm{D}-\mathrm{H} 5 \mathrm{D} 1$

$\mathrm{N} 2 \mathrm{D}-\mathrm{C} 5 \mathrm{D}-\mathrm{H} 5 \mathrm{D} 2$

C1D ${ }^{\mathrm{ii}}-\mathrm{C} 5 \mathrm{D}-\mathrm{H} 5 \mathrm{D} 2$

H5D1-C5D-H5D2

$\mathrm{H} 1 \mathrm{O} 1-\mathrm{O} 1 \mathrm{~W}-\mathrm{H} 2 \mathrm{O} 1$

$\mathrm{C} 2 \mathrm{C}-\mathrm{N} 1 \mathrm{C}-\mathrm{C} 1 \mathrm{C}-\mathrm{C} 5 \mathrm{C}^{\mathrm{i}}$

$\mathrm{C} 1 \mathrm{C}-\mathrm{N} 1 \mathrm{C}-\mathrm{C} 2 \mathrm{C}-\mathrm{C} 3 \mathrm{C}$

$\mathrm{N} 1 \mathrm{C}-\mathrm{C} 2 \mathrm{C}-\mathrm{C} 3 \mathrm{C}-\mathrm{C} 4 \mathrm{C}$

$\mathrm{C} 2 \mathrm{C}-\mathrm{C} 3 \mathrm{C}-\mathrm{C} 4 \mathrm{C}-\mathrm{N} 2 \mathrm{C}$

$\mathrm{C} 5 \mathrm{C}-\mathrm{N} 2 \mathrm{C}-\mathrm{C} 4 \mathrm{C}-\mathrm{C} 3 \mathrm{C}$

$\mathrm{C} 4 \mathrm{C}-\mathrm{N} 2 \mathrm{C}-\mathrm{C} 5 \mathrm{C}-\mathrm{C} 1 \mathrm{C}^{\mathrm{i}}$

$\mathrm{C} 3 \mathrm{D}-\mathrm{N} 1 \mathrm{D}-\mathrm{C} 2 \mathrm{D}-\mathrm{C} 1 \mathrm{D}$

$\mathrm{C} 5 \mathrm{D}^{\mathrm{ii}}-\mathrm{C} 1 \mathrm{D}-\mathrm{C} 2 \mathrm{D}-\mathrm{N} 1 \mathrm{D}$

$\mathrm{C} 2 \mathrm{D}-\mathrm{N} 1 \mathrm{D}-\mathrm{C} 3 \mathrm{D}-\mathrm{C} 4 \mathrm{D}$

$\mathrm{N} 1 \mathrm{D}-\mathrm{C} 3 \mathrm{D}-\mathrm{C} 4 \mathrm{D}-\mathrm{N} 2 \mathrm{D}$

$\mathrm{C} 5 \mathrm{D}-\mathrm{N} 2 \mathrm{D}-\mathrm{C} 4 \mathrm{D}-\mathrm{C} 3 \mathrm{D}$

$\mathrm{C} 4 \mathrm{D}-\mathrm{N} 2 \mathrm{D}-\mathrm{C} 5 \mathrm{D}-\mathrm{C} 1 \mathrm{D}^{\mathrm{ii}}$
109.1

109.1

107.8

$115.92(19)$

108.3

108.3

108.3

108.3

107.4

$106(3)$

$177.91(19)$

$-175.00(19)$

$55.8(3)$

$56.8(3)$

$-173.51(18)$

$65.9(2)$

$-173.36(19)$

73.5 (3)

$57.5(3)$

$55.5(3)$

$-172.66(19)$

$174.65(19)$

Symmetry codes: (i) $-x+2,-y+1,-z$; (ii) $-x+1,-y+1,-z+1$.

Hydrogen-bond geometry $\left(A,{ }^{\circ}\right)$

\begin{tabular}{lllll}
\hline$D-\mathrm{H} \cdots A$ & $D-\mathrm{H}$ & $\mathrm{H} \cdots A$ & $D \cdots A$ & $D-\mathrm{H} \cdots A$ \\
\hline $\mathrm{N} 1 C-\mathrm{H} 1 N C \cdots \mathrm{O} 2 B^{\mathrm{iii}}$ & 0.91 & 2.45 & $3.091(3)$ & 128 \\
$\mathrm{~N} 1 D-\mathrm{H} 1 N D \cdots \mathrm{O} 6 A$ & 0.91 & 2.38 & $3.140(3)$ & 142 \\
$\mathrm{~N} 1 D-\mathrm{H} 1 N D^{\cdots} \mathrm{O} 7 A$ & 0.91 & 2.16 & $2.942(3)$ & 143 \\
$\mathrm{~N} 1 D-\mathrm{H} 2 N D^{\cdots} \mathrm{O} 6 B^{\mathrm{ii}}$ & 0.91 & 1.87 & $2.768(3)$ & 169 \\
$\mathrm{~N} 2 C-\mathrm{H} 3 N C \cdots \mathrm{O} 6 A^{\mathrm{iv}}$ & 0.91 & 2.62 & $3.074(3)$ & 112 \\
$\mathrm{~N} 2 C-\mathrm{H} 4 N C \cdots \mathrm{O} 7 B^{\mathrm{v}}$ & 0.91 & 2.42 & $3.047(3)$ & 126 \\
$\mathrm{~N} 2 C-\mathrm{H} 4 N C \cdots \mathrm{O} 2 A$ & 0.91 & 2.52 & $3.200(3)$ & 132 \\
$\mathrm{~N} 2 D-\mathrm{H} 3 N D \cdots \mathrm{O} 6 B^{\mathrm{ii}}$ & 0.91 & 2.42 & $3.198(3)$ & 144 \\
$\mathrm{~N} 2 D-\mathrm{H} 4 N D \cdots \mathrm{O} 2 B^{\mathrm{ii}}$ & 0.91 & 2.65 & $3.357(3)$ & 136 \\
$\mathrm{O} 1 W-\mathrm{H} 1 O 1 \cdots \mathrm{O} 5 A^{\mathrm{ii}}$ & $0.84(1)$ & $2.38(10)$ & $2.999(3)$ & $130(11)$ \\
$\mathrm{O} 1 W-\mathrm{H} 2 O 1 \cdots \mathrm{O} 1 B$ & $0.84(1)$ & $2.05(4)$ & $2.774(3)$ & $143(5)$
\end{tabular}

Symmetry codes: (ii) $-x+1,-y+1,-z+1$; (iii) $x+1, y, z$; (iv) $x,-y+1 / 2, z-1 / 2$; (v) $-x+1, y-1 / 2,-z+1 / 2$; (vi) $-x+2, y+1 / 2,-z+1 / 2$. 